dence cited above that such secretion may be facilitated by protection from PERPP. Similarly, increased intake of $\omega-3$ fatty acids has been shown to result in a shift from small, dense LDLs to large, buoyant LDLs (14). This effect is likely to be multifactorial, with an important role for reduced cholesteryl ester transfer proteinmediated transfer of triglyceride to LDLs and subsequent lipolysis $(10,13)$. However, it is also consistent with evidence that larger, triglyceride-rich VLDLs are metabolic precursors of smaller LDL particles (10), and with the findings of Pan et al. (5) that increased PERPP reduces hepatic output of particles in this pathway (Figure 1).

A possible role for oxidation products in modulating hepatic lipoprotein secretion in humans can be assessed by determination of whether antioxidant treatment increases plasma transport or concentrations of ApoB-containing lipoproteins. In a randomized placebo-controlled study of 20,536 adults with pre-existing vascular disease or diabetes, daily supplementation with $600 \mathrm{mg}$ vitamin E, $250 \mathrm{mg}$ vitamin C, and $20 \mathrm{mg} \beta$-carotene resulted in small but statistically significant increases in triglyceride (11\%), ApoB (5\%), and LDL cholesterol (3\%), along with a small reduction in HDL cholesterol (15). No changes in triglyceride or LDL levels following antioxidant supplementation were found in a much smaller trial, but there was a reduction in the HDL2 cholesterol fraction, which was associated with reduced benefit on coronary disease endpoints when antioxidants were combined with simvastatin plus niacin therapy (16). Thus, although there is considerable evidence for the involvement of oxidative stress in many disease processes, including atherosclerosis, the potential for unintended outcomes of antioxidant therapy should serve as a warning against proceeding with such treatment in the absence of clinicaltrial evidence for benefit and safety.

Address correspondence to: Ronald M. Krauss, Children's Hospital Oakland Research Institute, 5700 Martin Luther King, Jr., Way, Oakland, California 94609, USA. Phone: (510) 450-7908; Fax: (510) 450-7909; E-mail: rmkrauss@lbl.gov.

1. Woollett, L.A., Spady, D.K., and Dietschy, J.M. 1992. Saturated and unsaturated fatty acids independently regulate low density lipoprotein receptor activity and production rate. J. Lipid Res. 33:77-88.

2. Kurushima, H., et al. 1995. Opposite effects on cholesterol metabolism and their mechanisms induced by dietary oleic acid and palmitic acid in hamsters. Biochim. Biophys. Acta. 1258:251-256.

3. Fisher, E.A., and Ginsberg, H.N. 2002. Complexity in the secretory pathway: the assembly and secretion of apolipoprotein B-containing lipoproteins. J. Biol. Chem. 277:17377-17380.

4. Fisher, E.A., et al. 2001. The triple threat to nascent apolipoprotein B. Evidence for multiple, distinct degradative pathways. J. Biol. Chem. 276:27855-27863.

5. Pan, M., et al. 2004. Lipid peroxidation and oxidant stress regulate hepatic apolipoprotein B degradation and VLDL production. J. Clin. Invest. 113:1277-1287. doi: $10.1172 / 200419197$.

6. Kris-Etherton, P.M., Harris, W.S., and Appel, L.J.
2003. Fish consumption, fish oil, omega-3 fatty acids, and cardiovascular disease. Arterioscler. Thromb. Vasc. Biol. 23:e20-e30.

7. Djousse, L., et al. 2003. Dietary linolenic acid is inversely associated with plasma triacylglycerol: the National Heart, Lung, and Blood Institute Family Heart Study. Am. J. Clin. Nutr. 78:1098-1102.

8. Mensink, R.P., Zock, P.L., Kester, A.D., and Katan, M.B. 2003. Effects of dietary fatty acids and carbohydrates on the ratio of serum total to HDL cholesterol and on serum lipids and lipoproteins: a meta-analysis of 60 controlled trials. Am. J. Clin. Nutr. 77:1146-1155.

9. Kummrow, E., Hussain, M.M., Pan, M., Marsh, J.B., and Fisher, E.A. 2002. Myristic acid increases dense lipoprotein secretion by inhibiting apoB degradation and triglyceride recruitment. J. Lipid Res. 43:2155-2163.

10. Berneis, K.K., and Krauss, R.M. 2002. Metabolic origins and clinical significance of LDL heterogeneity. J. Lipid Res. 43:1363-1379.

11. Dreon, D.M., et al. 1998. Change in dietary saturated fat intake is correlated with change in mass of large low-density-lipoprotein particles in men. Am. J. Clin. Nutr. 67:828-836.

12. Krauss, R.M., Lindgren, F.T., and Ray, R.M. 1980. Interrelationships among subgroups of serum lipoproteins in normal human subjects. Clin. Chim. Acta. 104:275-290.

13. Packard, C.J., et al. 2000. Apolipoprotein B metabolism and the distribution of VLDL and LDL subfractions. J. Lipid Res. 41:305-318.

14. Calabresi, L., Donati, D., Pazzucconi, F., Sirtori, C.R., and Franceschini, G. 2000. Omacor in familial combined hyperlipidemia: effects on lipids and low density lipoprotein subclasses. Atherosclerosis. 148:387-396.

15. Heart Protection Study Collaborative Group. 2002. MRC/BHF Heart Protection Study of antioxidant vitamin supplementation in 20,536 high-risk individuals: a randomised placebo-controlled trial. Lancet. 360:23-33.

16. Brown, B.G., et al. 2001. Simvastatin and niacin, antioxidant vitamins, or the combination for the prevention of coronary disease. N. Engl. J. Med. $345: 1583-1592$

\title{
Dysbindin-1 and schizophrenia: from genetics to neuropathology
}

\author{
Michael J. Owen, Nigel M. Williams, and Michael C. O'Donovan
}

Department of Psychological Medicine, University of Wales College of Medicine, Cardiff, United Kingdom.

\begin{abstract}
The gene encoding dysbindin-1 has recently been implicated in susceptibility to schizophrenia. In this issue of the JCI, Talbot et al. show that, contrary to expectations, dysbindin-1 is located presynaptically in glutamatergic neurons and is reduced at these locations in schizophrenia (see the related article beginning on page 1353). Further studies of dysbindin-1 and the proteins with which it interacts can be expected to throw light on the pathogenesis of schizophrenia.
\end{abstract}

Nonstandard abbreviations used: hippocampal formation (HF).

Conflict of interest: The authors have declared that no conflict of interest exists.

Citation for this article: J. Clin. Invest. 113:1255-1257 (2004). doi:10.1172/JCI200421470.
Schizophrenia is a common, severely disabling, mental disorder (1), and understanding its etiology and pathogenesis is one of the most important challenges facing psychiatry. Despite great endeavor, achieving this has proven difficult given the absence of a diagnostic neuropathology or biological markers, and few clear insights have emerged. However, there is currently a consensus that the disorder is, at least in part, neurodevelopmental (2). At the structural level, there are reductions in the neuropil and neuronal size that are widespread but not uniform, with temporal lobe structures, notably the hippocampal formation (HF), particularly affected (3). These changes in turn probably result from alterations in synaptic, dendritic, and axonal organization (3). At the functional level, accumulating evidence also implicates altered glutamate 


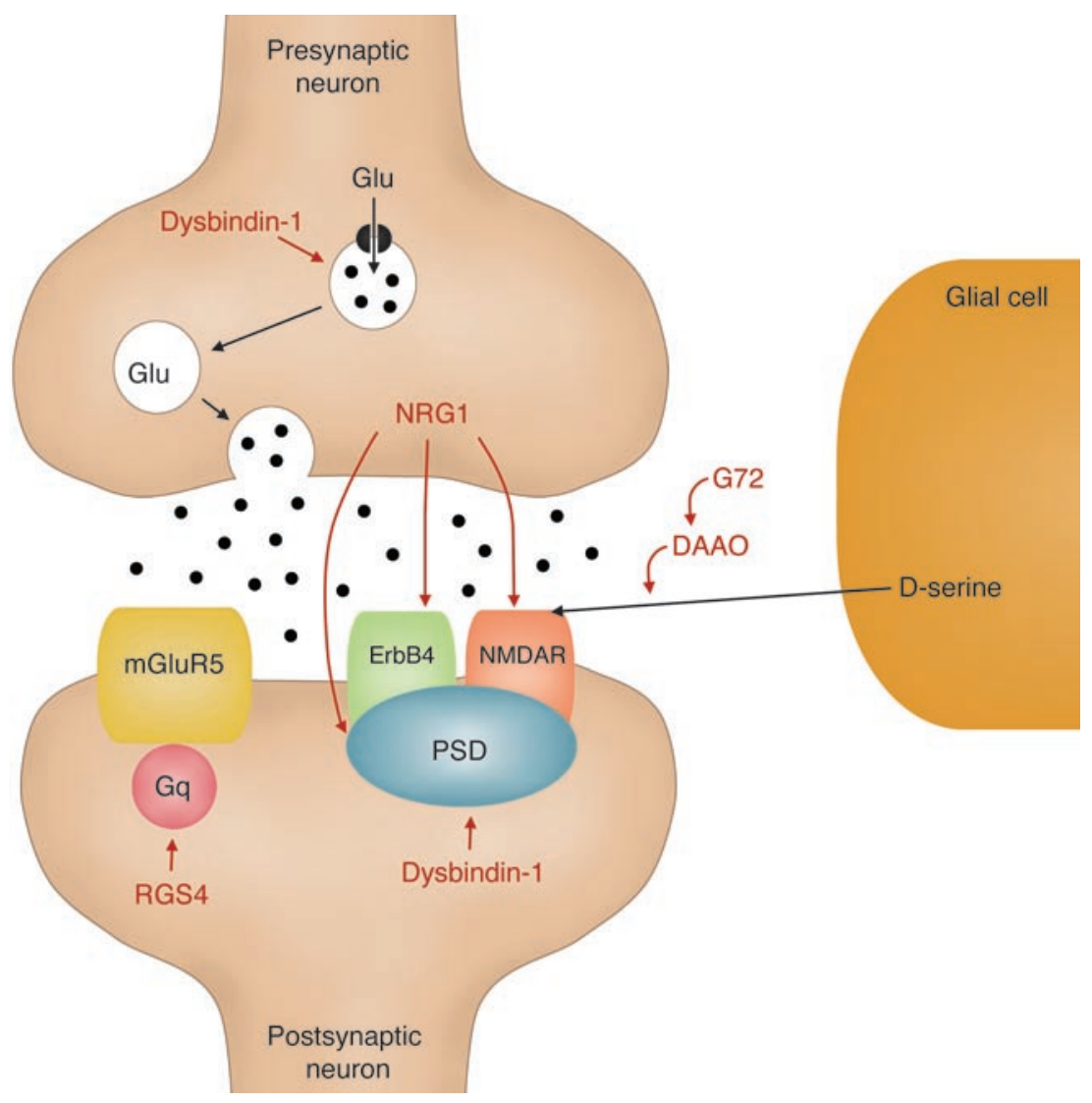

Figure 1

Schematic representation of a glutamatergic synapse. Several genes have been implicated in susceptibility to schizophrenia that can potentially impact on glutamate (Glu) synaptic function including dysbindin-1, neuregulin 1 (NRG1), G72, D-amino acid oxidase (DAAO), and regulator of $\mathrm{G}$ protein signalling 4 (RGS4). Dysbindin-1 may influence VGlutT-1 expression, synthesis, or degradation and is a component of the postsynaptic density (PSD). NRG1 is present in glutamate synaptic vesicles, regulates expression of $N$-methyl-D-aspartate receptors (NMDARs), activates ErbB4 receptors, which colocalize with NMDARs, and interacts with the PSD. G72 interacts with DAAO, which oxidizes D-serine, an endogenous modulator of NMDARs. RGS4 is a negative regulator of $G$ protein-coupled receptors, especially the metabotropic glutamate receptor 5 (mGluR5), via its effects on the G protein Gq. Figure modified with permission from The Lancet (7).

neurotransmission in addition to classical hyperdopaminergic explanations (4).

The most robust body of evidence regarding etiology comes from genetic epidemiological studies, which show that individual differences in liability are predominantly genetic, with heritability estimates around $80 \%$ (5). The most common mode of transmission is probably oligogenic, polygenic, or a mixture of the two (5), but the number of loci, the disease risk conferred by each, the extent of genetic heterogeneity, and the degree of interaction among loci are unknown. As for other common genetic disorders, the small effect on susceptibility conferred by any given locus makes identifying susceptibility genes by positional phin glycoprotein complex (11). The dystrophin complex is found in the sarcolemma of muscle (11) but is also located in postsynaptic densities in a number of brain areas (12). Although its functions are largely unknown, its location initially suggested that genetic variation in DTNBP1 might confer risk of schizophrenia by mediating effects on postsynaptic structure and function (8).

Despite the strong evidence implicating $D T N B P 1$, in the absence of schizophreniaassociated changes that alter the amino acid sequence of dysbindin-1, the actual susceptibility variants remain unknown. It even remains formally possible that these lie within an adjacent gene, but it is more likely that variation within DTNBP1 affects mRNA expression or processing. The latter possibility is indirectly supported by evidence for as yet unknown cis-acting polymorphisms affecting DTNBP1 expression in human brain (13).

\section{Presynaptic reductions of dysbindin-1 in schizophrenia}

In this issue of the JCI, Talbot and colleagues have tested this hypothesis by examining dysbindin-1 protein levels in human brain (14). Their findings provide several novel and important insights. First, while they confirmed fairly widespread neuronal distribution of dysbindin-1 and $\beta$-dystrobrevin, they also showed presynaptic localization of dysbindin-1 but not $\beta$-dystrobrevin, suggesting that dysbindin- 1 plays a presynaptic role in the HF that is independent of $\beta$-dystrobrevin and the dystrophin glycoprotein complex. Second, they found high levels of dysbindin-1 in the cells providing the intrinsic glutamatergic pathways of the HF and an inverse correlation with VGlutT-1, the main vesicular glutamate transporter present in the HF. This supports a relationship between dysbindin-1 and glutamate neurotransmission and suggests an effect on VGlutT-1 expression, synthesis, or degradation in the HF. Third, they found a significant reduction in dysbindin-1 expression at these hippocampal presynaptic sites in two separate populations of patients with schizophrenia. If correct, these findings suggest that, in the HF at least, dysbindin-1 may influence schizophrenia risk through presynaptic mechanisms that are independent of the dystrophin glycoprotein complex.

The authors have tried hard, by careful matching of cases and controls, to minimize the potential impact of many of the possible confounding variables that bedevil 
studies of postmortem brain. Most patients with schizophrenia have taken psychotropic medication for many years, and postmortem material from drug-naive patients is extremely difficult to obtain. Talbot and colleagues showed that dysbindin-1 and VGlutT-1 levels were not correlated with antipsychotic drug dose in the month before death and provide evidence against an effect of chronic drug treatment from studies of chronic haloperidol administration to mice (14). However, as the authors acknowledge, the validity of drug-treated mice as a control is open to question, and also no mention is made of matching of cases and controls for agonal state. Given the difficulties confirming previous postmortem findings in schizophrenia, it is now imperative that multiple independent replications be obtained. From the genetic perspective, it would also strengthen the mechanistic case of Talbot and colleagues if disease-associated single nucleotide polymorphisms or haplotypes can be linked to effects on gene expression. Here, ethnic variation in risk haplotypes and possible allelic heterogeneity within populations might prove problematic.

\section{Dysbindin-1 and the pathogenesis of schizophrenia}

These findings raise a number of important issues. First, what is the presynaptic function of dysbindin-1? A possible clue comes from recent evidence implicating it as a member of a protein complex involved in the trafficking of lysosomerelated organelles in association with the proteins muted, pallidin, and cappuccino (15). As Talbot et al. point out, lysosomes are found presynaptically in the $\mathrm{HF}$, and reduced lysosomal trafficking might result in elevated VGlutT-1 (14). However, further studies are required to identify dysbindin-1 interactors and to understand the control of DTNBP1 expression in brain. In addition, analysis of the mouse mutant sandy $(s d y)$, which, in its homozygous form, expresses no dysbindin-1 protein due to a large deletion in Dtnbp1, and of conditional knockouts might also be expected to throw light upon the function of dysbindin- 1 in brain. Second, Talbot and colleagues studied a restricted number of brain regions, particularly the HF. Dysbindin-1 is widely expressed in brain (11), and many lines of evidence suggest widespread involvement of different brain areas in the structural and functional abnormalities of schizophrenia (3). There is now a need to determine whether reduced dysbindin-1 levels are found in other brain areas and whether the association with glutamate is generalized or specific to the relatively restricted population of hippocampal regions implicated by Talbot and colleagues. Interestingly, Talbot and colleagues report regional variation in the distribution of the two dysbindin isoforms studied: the HF and cerebral cortex contained both 50 and 40 $\mathrm{kDa}$ isoforms while the cerebellar cortex contained only the $50 \mathrm{kDa}$ variant, implying that there are differences in their transcriptional control.

The identification of a potential role for dysbindin- 1 in glutamate transmission is of considerable interest. Evidence for glutamate dysfunction in schizophrenia is accumulating from both basic and clinical research (4). Moreover, a case can be made that other susceptibility genes identified on the basis of positional genetic studies, such as those encoding Neuregulin 1, G72, D-amino acid oxidase, and regulator of $G$ protein signalling 4, may have convergent effects upon glutamate synapses (Figure 1 ), although the links are speculative and several caveats remain (7).

Finally, if these findings are confirmed, they will indicate the potential power of positional genetics to home in on novel mechanisms. There are other linkages in schizophrenia that are as strong as that to $6 \mathrm{p} 22.3$, indicating the location of other susceptibility genes (6). On the basis of Talbot and colleagues' work (14) we can expect their identification to yield further crucial advances.
Address correspondence to: Michael J. Owen, Department of Psychological Medicine, Henry Wellcome Building, University of Wales College of Medicine, Heath Park, Cardiff CF14 4XN, United Kingdom. Phone: 44-920 7432 48; Fax: 44-920 7465 54; E-mail: owenmj@cf.ac.uk.

1. Gottesman, I.I. 1991. Schizophrenia genesis: the origins of madness. Freeman. New York, New York, USA. 296 pp.

2. Weinberger, D.R. 1995. From neuropathology to neurodevelopment. Lancet. 346:552-557.

3. Harrison, P.J. 1999. The neuropathology of schizophrenia. A critical review of the data and their interpretation. Brain. 122:593-624.

4. Moghaddam, B. 2003. Bringing order to the glutamate chaos in schizophrenia. Neuron. 40:881-884.

5. Owen, M.J., O’Donovan, M.C., and Gottesman, I.I. 2002. Schizophrenia. In Psychiatric genetics and genomics. P. McGuffin, M.J. Owen, and I.I. Gottesman, editors. Oxford University Press. New York, New York, USA. 247-266.

6. O'Donovan, M.C., Williams, N.M., and Owen, M.J. 2003. Recent advances in the genetics of schizophrenia. Hum. Mol. Genet. 12:R125-R133.

7. Harrison, P.J., and Owen, M.J. 2003. Genes for schizophrenia? Recent findings and their pathological implications. Lancet. 361:417-419.

8. Straub, R.E., et al. 2002. Genetic variation in the 6p22.3 gene DTNBP1, the human ortholog of the mouse dysbindin gene, is associated with schizophrenia. Am. J. Hum. Genet. 71:337-348.

9. Schwab, S.G., et al. 2003. Support for association of schizophrenia with genetic variation in the $6 \mathrm{p} 22.3$ gene, dysbindin, in sib-pair families with linkage and in an additional sample of triad families. Am. J. Hum. Genet. 72:185-190.

10. Williams, N.M., et al. 2004. Identification in two independent samples of a novel schizophrenia risk haplotype of the dystrobrevin binding protein gene (DTNBP1). Arch. Gen. Psychiatry. 61:336-344.

11. Benson, M.A., Newey, S.E., Martin-Rendon, E., Hawkes, R., and Blake, D.J. 2001. Dysbindin, a novel coiled-coil-containing protein that interacts with the dystrobrevins in muscle and brain. J. Biol. Chem. 276:24232-24241.

12. Blake, D.J., Hawkes, R., Benson, M.A., and Beesley, P.W. 1999. Different dystrophin-like complexes are expressed in neurons and glia. J. Cell Biol. 147:645-657

13. Bray, N.J., Buckland, P.R., Owen, M.J., and O'Donovan, M.C. 2003. Cis-acting variation in the expression of a high proportion of genes in human brain. Human Genet. 113:149-153.

14. Talbot, K., et al. 2004. Dysbindin-1 is reduced in intrinsic, glutamatergic terminals of the hippocampal formation in schizophrenia. J. Clin. Invest. 113:1353-1363. doi:10.1172/JCI200420425.

15. Li, W., et al. 2003. Hermansky-Pudlak syndrome type 7 (HPS-7) results from mutant dysbindin, a member of the biogenesis of lysosome-related organelles complex 1 (BLOC-1). Nature Genet. 35:84-89. 